\title{
Can somatostatin antagonism prevent hypoglycaemia during exercise in type 1 diabetes?
}

\author{
Nadine Taleb $^{1} \cdot$ Rémi Rabasa-Lhoret $^{1,2}$
}

Received: 7 April 2016 / Accepted: 15 April 2016/Published online: 6 May 2016

(C) Springer-Verlag Berlin Heidelberg 2016

\begin{abstract}
The prevention and management of exerciseinduced hypoglycaemia remains a challenge for patients with type 1 diabetes. Strategies involving changes to insulin dosing and/or carbohydrate consumption in anticipation of or during different types of exercise have proved to be helpful but not sufficient to fully prevent the hypoglycaemic risk. Meanwhile, the defect in glucagon secretion in response to hypoglycaemia in diabetes and the contributory role of somatostatin to this dysregulation constitute an important therapeutic target. In this issue of Diabetologia (DOI 10.1007/s00125-016-39530 ), Leclair et al show that selective somatostatin receptor 2 antagonists can enhance glucagon secretion in rats with streptozotocin-induced diabetes during exercise. The implications of their interesting findings are discussed, as well as limitations and potential for clinical applications, together with other glucagon-based options for tackling exercise-induced hypoglycaemia in diabetes.
\end{abstract}

Keywords Exercise - Glucagon · Hypoglycaemia Somatostatin receptor type 2 antagonists · Type 1 diabetes

$\begin{array}{ll}\text { Abbreviations } \\ \text { AP } & \text { Artificial pancreas } \\ \text { SSTR2 } & \text { Somatostatin receptor } 2 \\ \text { SSTR2a } & \text { Somatostatin receptor } 2 \text { antagonist }\end{array}$

Rémi Rabasa-Lhoret

Remi.rabasa-lhoret@ircm.qc.ca

1 Institut de recherches cliniques de Montréal, 110, avenue des Pins Ouest, Montréal, Québec H2W 1R7, Canada

2 Nutrition Department, Faculty of Medicine, Université de Montréal, Montréal, Québec, Canada
For patients with type 1 diabetes, hypoglycaemia constitutes a major hindrance to achieving optimal glucose control [1]. While often underestimated, the burden of hypoglycaemia weighs heavily on a patient's life at the physical (e.g. fall, coma or even death) as well as emotional levels (e.g. fear, anxiety) [1]. Moreover, recurrent hypoglycaemia leads to a vicious cycle of hypoglycaemia unawareness with increased severity and frequency of hypoglycaemic events [1]. Not surprisingly then, fear of hypoglycaemia is identified as the major barrier to exercise, which in turn entails an elevated risk of its occurrence [2]. Regular physical activity is an important component of type 1 diabetes owing to its several established health benefits: it improves well-being, cardiovascular risk profile and is associated with an overall lower mortality risk [3]. Nevertheless, the majority of patients with type 1 diabetes adopt a sedentary lifestyle which, similar to the general population, is associated with a poorer cardiometabolic profile $[4,5]$. Even in the context of modern treatment modalities, the prevention and management of exercise-related hypoglycaemia remains a big challenge to patients with type 1 diabetes and their healthcare professionals.

Physical activity can be undertaken in multiple forms (aerobic, resistance, interval, etc.), intensities and durations, each triggering distinct physiological responses. In nondiabetic individuals, coordinated hormonal responses ensure the maintenance of normal glycaemic ranges; the rate of muscular glucose uptake (during and after exercise) is matched with proportional fuel supplies from hepatic glucose production $[1,3]$. The key to glycaemic control is adequate portal insulin:glucagon ratios in addition to contributions from other counterregulatory hormones (growth hormone, catecholamines, growth hormone and cortisol). Individuals with type 1 diabetes are neither able to suppress the subcutaneously delivered insulin nor stimulate an adequate response to hypoglycaemia via the main counterregulatory hormones 
(glucagon and catecholamines) [1, 3]. Patients face the challenge of compensating for their dysregulated hormonal responses by adjusting two key factors: insulin dosing and/ or carbohydrate consumption. Adjustments are directed by several complex determinants such as exercise type, intensity, duration and meal timing in addition to the specific insulin regimen used (multiple injections vs insulin pump) and physical fitness etc. (Fig. 1). The management strategies proposed to patients are feasible but still fall short of fully preventing early and late exercise-related hypoglycaemia. In addition, they require a lot of anticipation and timely adjustments.

The problem can, however, be approached from another perspective targeting the defective secretion of the counterregulatory hormones. Glucagon makes an excellent therapeutic candidate during exercise, being the main counterregulatory hormone to insulin and hypoglycaemia. Interestingly in diabetes, glucagon is dual-faceted and can be therapeutically targeted in opposite directions depending on the context of its use; it can be either suppressed (e.g. to improve postprandial glucose excursions) or stimulated (e.g. to prevent hypoglycaemia and/or modulate food intake). As such, drugs for both glucagon antagonism and agonism (e.g. GLP-1/glucagon co-agonists) are being developed.

Somatostatin is a long known suppressor of several hormones but its specific contributory role to the defective glucagon secretion in diabetes has recently been characterised [6]. It exerts an intra-islet paracrine effect on alpha cells through the highly expressed somatostatin type 2 receptor
(SSTR2). A selective SSTR2 antagonist (SSTR2a) was shown to improve glucagon response in hypoglycaemic clamps and recurrent hypoglycaemic events in resting diabetic rats [7].

Leclair et al then took the lead and examined the effect of intraperitoneal injections of PRL-2903, a selective SSTR2a, on glucagon counterregulation and glucose profile during exercise in a rodent model of type 1 diabetes. Their interesting work is reported in the current issue of Diabetologia [8]. Streptozotocin-induced diabetic rats (a widely used model of type 1 diabetes), habituated to treadmill exercise, were studied during $30 \mathrm{~min}$ of continuous exercise followed by a $60 \mathrm{~min}$ recovery period. An insulin bolus with no concomitant feeding aiming for a blood glucose level of $5 \mathrm{mmol} / \mathrm{l}$ was injected an hour prior to exercise to reproduce the state of hyperinsulinaemia frequently encountered by patients with type 1 diabetes. The protocol consisted of a crossover design of two types of intervention: SSTR2a injections (PRL-2903, $10 \mathrm{mg} / \mathrm{kg}$ i.p. $)$ or saline $(154 \mathrm{mmol} / \mathrm{l} \mathrm{NaCl})$ injection. SSTR2a-treated rats had a three- to sixfold increase in their glucagon levels in response to exercise $(10 \%$ of rats were deemed non-responders on secondary analysis) [8]. They could also maintain higher glucose levels when treated with SSTR2a in comparison with saline most of the time. However, despite the rise in glucagon levels, hypoglycaemia still occurred towards the end of exercise $(30 \mathrm{~min})$ with SSTR2a treatment. Unfortunately, insulin levels were not measured, limiting the interpretation of the data. It is possible that higher than expected insulin levels could explain these hypoglycaemic episodes. Indeed, insulin boluses given

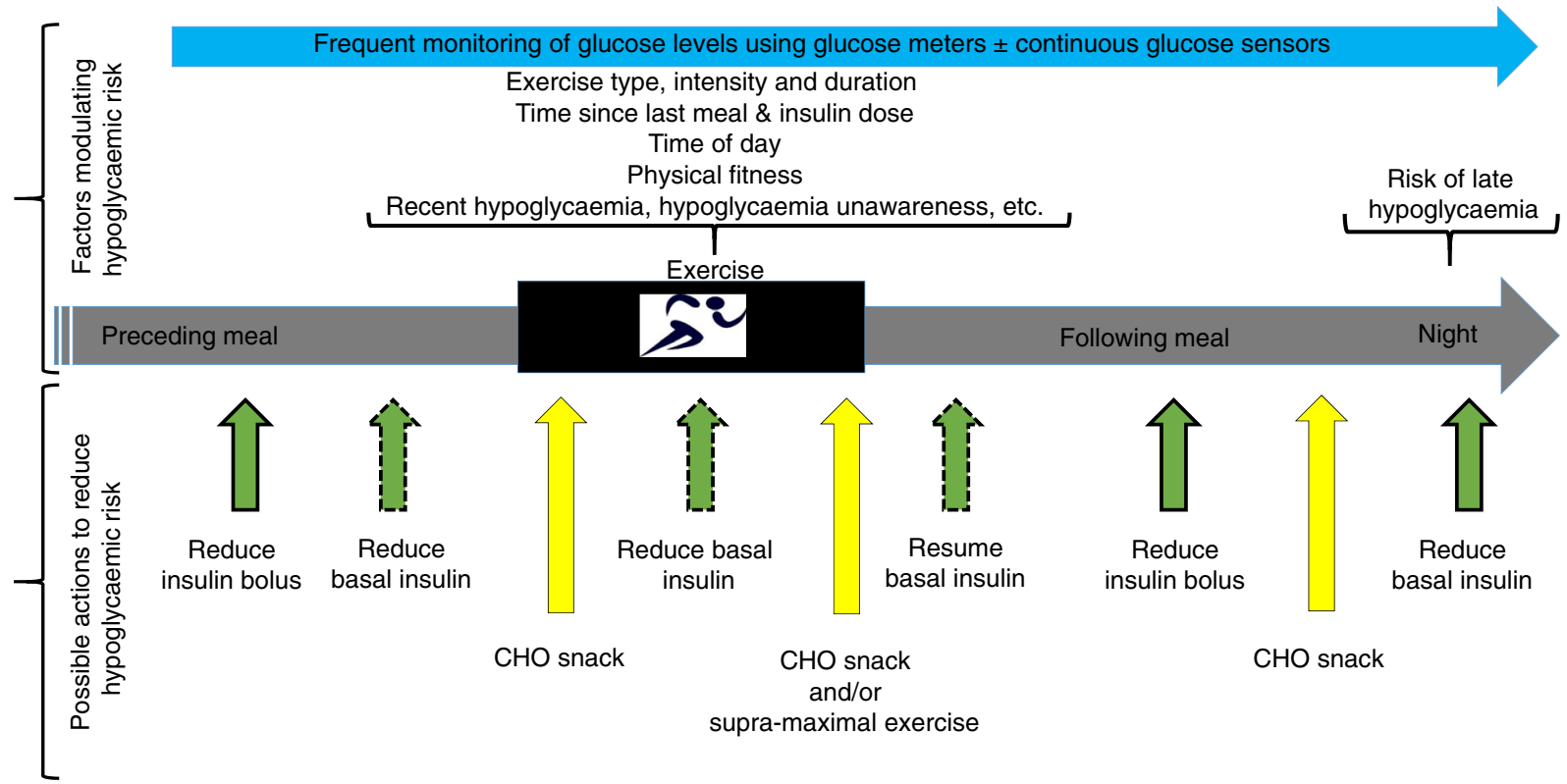

Fig. 1 The complexity of exercise-related hypoglycaemia prevention in type 1 diabetes: modulating factors and possible actions on insulin dosing and carbohydrate $(\mathrm{CHO})$ intake. The percentage of insulin dose reduction depends on the factors modulating hypoglycaemic risk and on the type of insulin regimen used; basal insulin reduction can be a dynamic process if an insulin pump is used (continuous subcutaneous insulin infusion [CSII]) but is only possible at specific times in the case of multiple daily injection regimens (MDI). The plain green arrows apply both to patients using MDI and CSII; dashed green arrows apply only to CSII users 
without feeding are expected to reach their peak levels at the end of exercise. This might explain the attenuated effect of induced endogenous glucagon secretion on reversing hypoglycaemia in SSTR2a-treated rats. Alternatively, the authors attribute this decreased effect to the lower hepatic glycogen stores seen in diabetic rats, despite insulin treatment, when compared with healthy littermates [8]. Future trials might be able to tease out the dominant mechanism that attenuates the effect of secreted glucagon in maintaining euglycaemia. Another potential drawback to the use of SSTR2a was the non-sustained induction of glucagon levels at $70 \mathrm{~min}$ post exercise when the glucose nadir was reached [8]. A dynamic communication exists among the different intra-islet cell types [9]. Glucagon has been reported to stimulate somatostatin and insulin release [10]; thus, the initially rising glucagon levels may attenuate the effect of SSTR2a by stimulating somatostatin release from delta cells and, to a lesser extent, insulin from some preserved beta cells from the streptozotocin rodent model. The exact mechanisms of the non-sustained effect of SSTR2a or possible tachyphylaxis through internalisation of the receptor itself need to be deciphered to enhance its function [11]. As the hypoglycaemic risk is increased not only during exercise but also over the following hours, even after glycogen stores are restored, a more prolonged SSTR2a effect could be extremely useful in preventing late onset (mainly nocturnal) hypoglycaemia.

SSTR2a has the major advantage of glucose sensitivity, exerting an effect only in states of hypoglycaemia, which makes it an attractive therapeutic option without significant hyperglycaemic risk. Safety will need to be proved, however, since SSTR2 is expressed in several tissues, mainly the stomach, adrenal medulla, cerebral cortex, hypothalamus and pituitary gland [11]. Potential adverse effects could thus include increased gastric acid secretion and effects on pituitary and adrenal hormone secretion, while potential central effects will depend on its ability to cross the blood-brain barrier. A detailed safety profile needs to be established before translation to human studies.

In addition to elevating endogenous glucagon levels through SSTR2a, exogenous glucagon can be used directly as a therapeutic option. Its only clinically approved indication to date is the $1 \mathrm{mg}$ subcutaneous dose, for immediate use after reconstitution, to treat severe hypoglycaemia. Simpler nasal and stable soluble glucagon formulations are undergoing rapid development for hypoglycaemia treatment. However, a novel therapeutic indication of exogenous glucagon has been proposed and is being tested in the development of artificial pancreas (AP) systems. AP operates by an automated closed loop of a continuous glucose sensor linked to an algorithm, which dynamically commands the rates of insulin (singlehormone AP) and glucagon (dual-hormone AP) infusion pumps. Glucagon is administered as mini-boluses not only to treat but also to prevent hypoglycaemia. A supplementary benefit of glucagon to further reduce hypoglycaemia was highlighted when dual- and single-hormone AP systems were directly compared $[12,13]$. Recent data indicate that, in the context of exercise, dual-hormone AP using glucagon is more efficacious than insulin-only AP in preventing hypoglycaemic events requiring treatment and keeping blood glucose within target levels [14]. Several steps are needed before dualhormone AP systems are approved: stable liquid glucagon formulations are needed for long-term use in the hormone infusion pumps, the safety of chronic use need to be established, and the added benefit of glucagon will need to be weighed against the increased complexity of its use. Alternatively, as demonstrated by Leclair et al [8], SSTR2 antagonism enhances endogenous glucagon secretion only during hypoglycaemia, possibly offering a more physiological option. Physical activity continues to prove its benefits and importance as an integral component in the management of all types of diabetes. Therefore, the more options that are tested and eventually made clinically available for use alone or in combination to target hypoglycaemia, the better.

Diabetes is increasingly recognised as a multihormonal disease afflicting a heterogeneous group of people. Consequently, the future of treatment of exercise-related hypoglycaemia in diabetes will probably head towards a personalised approach. This will depend on the availability of wide-ranging options to choose what best fits a patient's needs, from insulin regimens and technological devices to different hormonal additions such as glucagon through replacement or induction by SSTR antagonism. In addition to exercise-induced hypoglycaemia in type 1 diabetes, hypoglycaemia is also problematic for many patients with advanced type 2 diabetes who have lost most of their beta cell function. Thus, SSTR2 antagonism could have wide applications in mitigating hypoglycaemic risk and warrants continued research efforts to develop its promising therapeutic uses.

Duality of interest RR-L has received consultant's or speaker's honoraria or grants from AstraZeneca, Becton Dickinson, Boehringer Ingelheim, Eli Lilly, Janssen, Lifescan, Medtronic, Merck, Novartis, Neomed, Novo Nordisk, Roche, Sanofi-Aventis, Takeda, and Valeant and owns intellectual property in the area of AP research.

NT declares no duality of interest associated with this manuscript.

Contribution statement Both RRL and NT drafted and approved the final submitted document.

\section{References}

1. Cryer PE (2008) The barrier of hypoglycemia in diabetes. Diabetes 57:3169-76

2. Brazeau A-S, Rabasa-Lhoret R, Irene S, Mircescu H (2008) Barriers to physical activity among patients with type 1 diabetes. Diabetes Care 31:2108-9 
3. Galassetti P, Riddell MC (2013) Exercise and type 1 diabetes (T1DM). Compr Physiol 3:1309-36

4. Brazeau AS, Leroux C, Mircescu H, Rabasa-Lhoret R (2012) Physical activity level and body composition among adults with type 1 diabetes. Diabet Med 29:e402-e408

5. Leroux C, Brazeau A-S, Gingras V, Desjardins K, Strychar I, Rabasa-Lhoret R (2014) Lifestyle and cardiometabolic risk in adults with type 1 diabetes: a review. Can J Diabetes 38:62-69

6. Karimian N, Qin T, Liang T, Osundiji M, Huang Y, Teich T et al (2013) Somatostatin receptor type 2 antagonism improves glucagon counterregulation in biobreeding diabetic rats. Diabetes 62: 2968-2977

7. Yue JTY, Riddell MC, Burdett E, Coy DH, Efendic S, Vranic M (2013) Amelioration of hypoglycemia via somatostatin receptor type 2 antagonism in recurrently hypoglycemic diabetic rats. Diabetes 62:2215-2222

8. Leclair E, Liggins RT, Peckett AJ (2016) Glucagon responses to exercise-induced hypoglycaemia are improved by somatostatin receptor type 2 antagonism in a rat model of diabetes. Diabetologia. doi:10.1007/s00125-016-3953-0

9. Brunicardi FC, Kleinman R, Moldovan S, Nguyen TH, Watt PC, Walsh J et al (2001) Immunoneutralization of somatostatin, insulin, and glucagon causes alterations in islet cell secretion in the isolated perfused human pancreas. Pancreas 23:302-308
10. Dolais-Kitabgi J, Freychet P (1979) Neurotensin inhibits glucose but not glucagon-induced insulin and somatostatin release in isolated islets. Diabete Metab 5:275-7

11. Yue JTY, Burdett E, Coy DH, Giacca A, Efendic S, Vranic M (2012) Somatostatin receptor type 2 antagonism improves glucagon and corticosterone counterregulatory responses to hypoglycemia in streptozotocin-induced diabetic rats. Diabetes 61:197-207

12. Haidar A, Legault L, Messier V, Mitre TM, Leroux C, Rabasalhoret R (2014) Comparison of dual-hormone artificial pancreas, single- hormone artificial pancreas, and conventional insulin pump therapy for glycaemic control in patients with type 1 diabetes: an open-label randomised controlled crossover trial. Lancet Diabetes Endocrinol 8587:1-10

13. Haidar A, Legault L, Matteau-Pelletier L, Messier V, Dallaire M, Ladouceur M et al (2015) Outpatient overnight glucose control with dual-hormone artificial pancreas, single-hormone artificial pancreas, or conventional insulin pump therapy in children and adolescents with type 1 diabetes: an open-label, randomised controlled trial. Lancet Diabetes Endocrinol 3:595-604

14. Taleb N, Haidar A, Suppere C, Emami A, Messier V, Legault L et al (2015) The efficacy of single-and dual-hormone artificial pancreas systems at regulating glucose levels during continuous and interval exercise in type 1 diabetes. Diabetes Am Diabetes Assoc 64:A271A272, abstract 